\title{
Dinâmica espaço-temporal da disseminação da COVID-19 em cidade localizada na fronteira Brasil-Uruguai
}

\author{
Dalvana Lopes Ribeiro ${ }^{1}$ \\ Programa de Pós-Graduação em Modelagem Computacional / FURG, Rio Grande, RS \\ André Andrade Longaray ${ }^{2}$ \\ Programa de Pós-Graduação em Modelagem Computacional / FURG, Rio Grande, RS
}

\begin{abstract}
Resumo. O objetivo deste trabalho consiste na avaliação da dinâmica e evolução espaço temporal da COVID-19 em Quaraí, fronteira oeste do Rio Grande do Sul. No desenvolvimento do trabalho utilizou-se a técnica de densidade de Kernel com um raio de 120 metros e tamanho de pixel igual a 4, para se obter uma boa resolução das concentrações de casos no perímetro urbano da cidade. Para avaliar a relação entre casos com comorbidades e a pontuação de sintomas foi realizado o teste de Moran. Com um índice de $\mathrm{I}=0,412$, houve correlação espacial global positiva e significativa $(\mathrm{p}=0,001)$ entre o número de casos com comorbidades e casos com pontuações elevadas. Além disso, o número de reprodução do vírus Sars-CoV-2 ao longo do período de estudo pode ser estimado. Para isso, foi considerado um período infeccioso médio de 4,7 dias com um desvio padrão de 2,9 dias baseado e uma janela de tempo de $\mathrm{w}=7$ dias. Os resultados mostram a espacialização da disseminação da doença nos bairros da cidade, com especial atenção aos bairros centrais e sul. Além disso, foi possível perceber que as flexibilizações de medidas de controle bem como a influência da fronteira com o Uruguai podem ter contribuído para o aumento de casos no fim de 2020. A aplicação de ferramentas espaciais são importantes aliadas no combate ao novo corona vírus.
\end{abstract}

Palavras-chave. COVID-19, Análise Espacial, Número de Reprodução, SARS-CoV-2.

\section{Introdução}

A pandemia causada pela doença de Coronavírus (COVID-19) tem se tornado uma emergência mundial e desafia as mais diversas sociedades. Com seu início no fim de dezembro de $2019 \mathrm{em}$ Wuhan, na China, o surto é considerado o propulsor de uma das maiores crises sanitárias já vistas [17].

No decorrer das investigações sua causa foi relacionada a um novo tipo de Coronavírus, o SARSCov-2 [7], que possui uma forte capacidade de infectar seres humanos podendo levar a morte em alguns casos [15]. Com isso, em janeiro de 2020 a Organização Mundial da Saúde (OMS) declarou emergência de saúde pública Internacional e em 11 de março foi alcançado o nível de pandemia. Desde então, a rápida ascensão da doença tem preocupado lideranças do mundo inteiro e assolando países.

Diante da presente situação, o que se observa são duas principais estratégias de enfrentamento: a busca por tratamentos médicos e a constante procura de soluções que reduzam a curva de contaminação da doença para que possa ser suportada pelo sistema de saúde. Nessa batalha, além da pesquisa médica e biológica, os estudos teóricos baseados em estatística ou em modelagem

\footnotetext{
${ }^{1}$ lopesribeirodalvana20@gmail.com.

2 andrelongaray@gmail.com.
} 
matemática e espacial também são tidos como importantes aliados [11]. A implementação desses estudos na situação atual pode auxiliar de várias formas, tanto na compreensão das características epidêmicas do surto, na previsão do ponto de inflexão como também podem servir no embasamento de medidas para coibir a disseminação.

A COVID-19 ainda envolve inúmeras incógnitas, e muitas delas têm uma dimensão espacial que leva a entender o fenômeno como geográfico e potencialmente mapeável [5]. Concernente a isso, inúmeras técnicas de geoprocessamento estão sendo usadas tanto no controle quanto na busca de possíveis características espaciais que possam surgir. Dentre os métodos mais utilizados a estimativa de Kernel e o teste de Moran usado em análises de correlações espaciais, tem se mostrado importantes ferramentas em tempos de pandemia [1]).

A estimativa da densidade do Kernel (KDE) é um método importante para mapear padrões espaciais de eventos pontuais e diversos estudos nas mais diferentes áreas fazem uso dessa ferramenta [18]. Já o teste de Moran avalia a covariância de um valor em uma localização de índice com a média dos valores de seus vizinhos [2]. Sendo assim, o objetivo desse estudo é analisar a dinâmica e evolução da COVID-19 a nível municipal utilizando as técnicas como estimativa de Kernel e o índice de Moran.

\section{Delineamento Metodológico}

Para o estudo foram analisadas cerca de 377 fichas de investigação provenientes da Secretaria Municipal de Saúde do município de Quaraí. Tais fichas continham informações tanto de cunho epidemiológico como dados usados no rastreamento da doença. Os shapefiles utilizados para o mapeamento e análises espaciais são oriundos do site do Instituto Brasileiro de Geografia e Estatística (IBGE). Já as informações referentes aos casos do departamento de Artigas, no Uruguai foram obtidas por meio dos informes nacionais disponibilizados pelo Sistema Nacional de Emergências. Para o processamento espacial foram utilizados os softwares QGis 3.10.7 e GeoDa. Já para a determinação do número de reprodução $(R)$ foi utilizado o pacote EpiEstim do $R$ Project.

\section{1 Área de Estudo}

O município de Quaraí está localizado na região sudoeste do Rio Grande do Sul distante cerca de 595 quilômetros da capital estadual, Porto Alegre. Com uma população de 22.607 habitantes (Instituto Brasileiro de Geografia e Estatística) e área de $3.148 \mathrm{~km}^{2}$, é um dos maiores municípios gaúchos em área territorial fazendo parte da Região da Campanha conforme Figura 1.

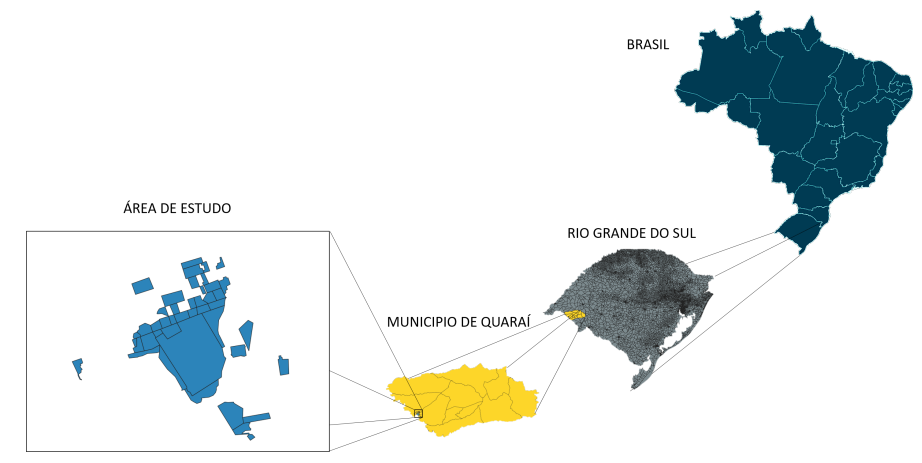

Figura 1: Área de Estudo.

A região faz parte da linha de fronteira, composta pelos municípios que fazem divisa com os países vizinhos, ao fazer fronteira com a cidade uruguaia de Artigas que possui aproximadamente 
40.658 habitantes (Instituto Nacional de Estatística do Uruguai) e a comunicação com Quaraí se dá pela Ponte Internacional da Concórdia.

\subsection{Análise Espacial}

\subsubsection{Estimativa de Kernel}

A estimativa de Kernel é uma técnica de interpolação que permite resultados satisfatórios em extração de pontos de acesso de grandes conjuntos de dados [6]. Consiste num método bem estabelecido de análise espacial baseado em testes não paramétricos [8], usado para identificação de padrões espaciais. O estimador de densidade de Kernel projeta uma vizinhança circular ao redor de cada ponto de determinada amostra, correspondendo ao raio de influência, onde então é aplicada a função de Kernel que pode ser calculada com a seguinte equação 1:

$$
\lambda_{R}(P)=\sum_{i=1}^{n} \frac{1}{R^{2}} K\left(\frac{P-P_{i}}{R}\right),
$$

onde $P$ representa uma localização qualquer em $R, K$ é uma função de Kernel bivariada e simétrica e $R>0$ é o raio de influência que também é conhecido como largura da banda. $\mathrm{O}$ valor da densidade de Kernel pode mudar com a distância do ponto central. Quanto mais próximo estiver o ponto central, maior será o valor da densidade e mais longe do centro ponto, menor será a densidade.

\subsection{2 Índice de Moran}

A autocorrelação espacial possibilita a mensuração da tendência de semelhança ou diferença de uma determinada região em relação as regiões vizinhas considerando um determinado atributo ou indicador característico. Dentre as medidas mais usadas está o índice de Moran que avalia a covariância de um valor em uma localização de índice com a média dos valores de seus vizinhos [2] e pode ser calculado pela equação 2

$$
I=\frac{n}{\sum_{i=1}^{n} \sum_{j=1}^{n} w_{i, j}} \frac{\sum_{i=1}^{n} \sum_{j=1}^{n} w_{i, j}\left(x_{i}-\bar{x}\right)\left(x_{j}-\bar{x}\right)}{\sum_{i=1}^{n}\left(x_{i}-\bar{x}\right)^{2}},
$$

onde $n$ é o número de regiões, $x_{i}$ valor do atributo na área $I, \bar{x}$ é a média do valor do atributo na região de estudo e $w_{i, j}$ elementos do valor do atributo na área $I$ (pesos espaciais da matriz $W$ ).

A autocorrelação espacial pode ser avaliada globalmente ou localmente. As medidas globais resumem a autocorrelação espacial, enquanto as medidas locais avaliam a autocorrelação espacial localizada dentro de um local de estudo [1]. Os indicadores locais de associação espacial (LISA) permitem a decomposição do $I$ de Moran global inferindo o escopo do agrupamento em áreas menores [12].

\subsection{Número de Reprodução $\left(R_{t}\right)$}

O número reprodutivo $\left(R_{t}\right)$ quantifica o número esperado de infecções secundárias causadas por uma única infecção Individual [14]. A variação de $R_{t}$ ao longo do tempo reflete a eficácia das medidas de controle e destaca quando os esforços de controle precisam ser intensificados. Neste trabalho o $R_{t}$ foi calculado com o pacote EpiEstim [3] baseado nos padrões estipulados por [10] para a COVID-19. Foi usado então, um período infeccioso médio de 4,7 dias com um desvio padrão de 2,9 dias baseado e uma janela de tempo de $\mathrm{w}=7$ dias .

\section{Resultados e Discussão}

Neste trabalho buscou-se encontrar o número de reprodução $\left(R_{t}\right)$ durante o período do estudo (Abril/2020 - Janeiro 2021) para verificar sua relação com as medidas adotadas bem com 
a dinâmica espacial do processo. A evolução espacial da COVID-19, o $R_{t}$ e o número de casos cumulativos podem ser observados na Figura 2

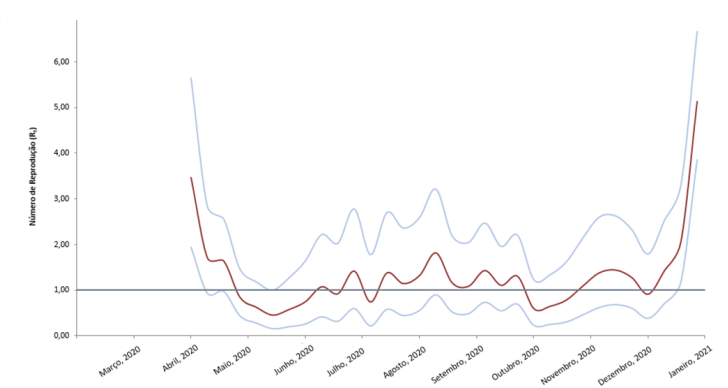

B

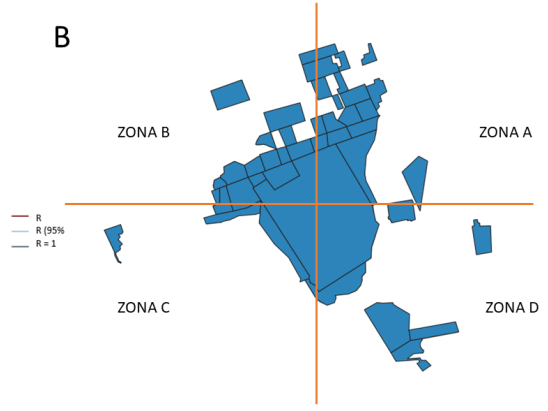

C
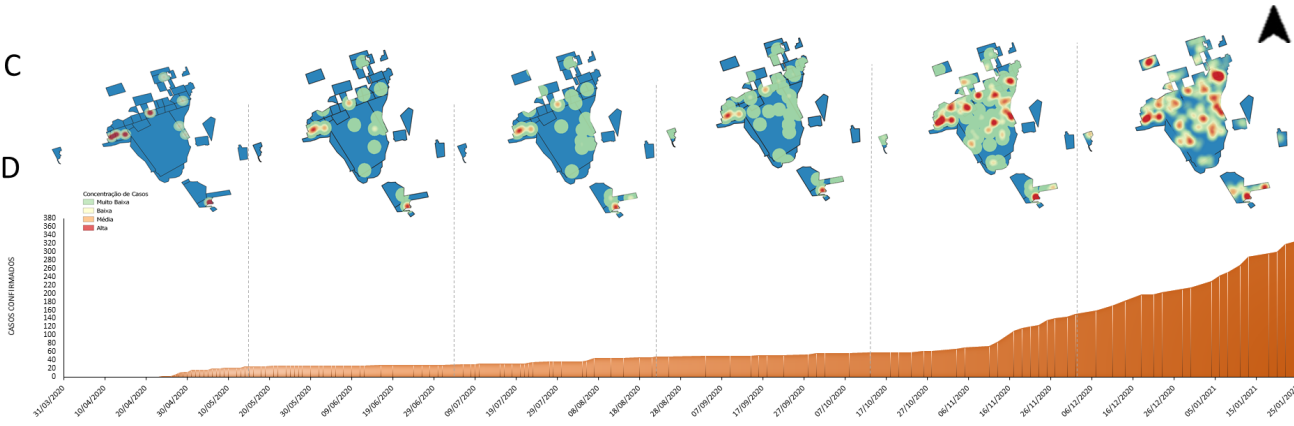

A

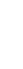

Figura 2: A - Número de Reprodução $\left(R_{t}\right)$ ao longo do período de estudo. B - Zonas de estudo. C Evolução espacial da COVID-19 entre os bairros de Quaraí. D - Casos cumulativos ao longo do período de estudo.

De acordo com a Figura 2 A-C é possível observar a dinâmica espacial dos casos e a variação do número de reprodução no decorrer do tempo. Inicialmente a contaminação ocorre em pontos isolados com um $R_{t}$ acima de 3 , entre a zona oeste e sul da cidade. Durante os meses maio e junho outros cinco focos da doença surgem em áreas diferentes. Apesar da redução do $R_{t}$ no fim de maio, os dois meses seguintes apresentam um aumento do número de casos praticamente nas mesmas áreas. Em novembro ocorre um aumento e espalhamento considerável dos casos. Esse comportamento continua no mês de dezembro e janeiro onde as áreas que continham concentrações médias evoluem para concentrações altas da doença e o $R_{t}$ ultrapassa seu limite inicial. Um dos fatores que pode ter contribuído para este aumento procedente de viagens. Cerca de $77 \%$ dos casos positivos que viajaram, tanto de forma intermunicipal quanto interestadual, ocorreram neste período.

Outra possível fonte de influência no aumento substancial de casos a partir de novembro pode estar associado ao efeito "fronteira". Quaraí faz fronteira com a cidade de Artigas no Uruguai. As duas cidades são separadas pela ponte internacional da Concórdia e ambas possuem uma relação muito próxima baseada na atividade econômica. As zonas fronteiriças tem especial importância, pois esforços multinacionais unidos podem conduzir boas estratégias para o enfrentamento desta crise [9].

A Figura 3 mostra um aumento no número de casos na cidade de Artigas em agosto, diminuindo em setembro e voltando a crescer em outubro. Além disso, pode-se observar uma alternância no comportamento da doença nas cidades entre os meses de novembro e dezembro porém uma ascensão 


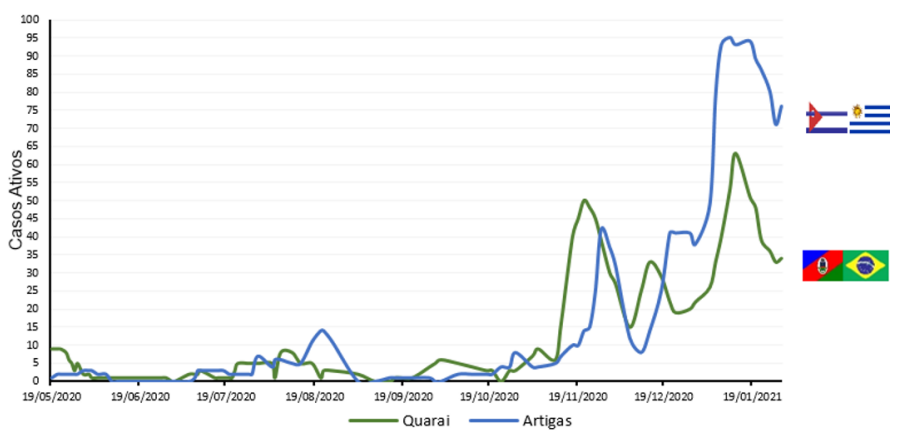

Figura 3: Relação de casos ativos de Quaraí e Artigas.

maior obtida pela cidade de Artigas a partir do fim de dezembro. Essas diferenças ocorrem devido ao sistema de enfrentamento diferente entre os dois países. O fluxo de mobilidade urbana que ocorre entre as duas cidades também pode ter influenciado a grande taxa de contaminação no fim de 2020.

Além do mapeamento dos casos, um dos objetivos deste trabalho foi avaliar a correlação espacial de casos com comorbidades e a pontuação dos sintomas. Acredita-se que a doença, em indivíduos com problemas de saúde ou comorbidades subjacentes, tenham uma progressão cada vez mais rápida e grave, muitas vezes levando à morte [13].

No Brasil, diversas secretarias de saúde fazem uso das fichas de investigação da COVID-19. As fichas correspondem a formulários aplicados a todos os indivíduos que apresentam sintomas relacionados a COVID-19. Cada sintoma possui uma determinada pontuação e a soma destes constituem o grau de risco que o indivíduo se encontra. Portanto, mediu-se a autocorrelação espacial bivariada entre os fatores: comorbidade e pontuação a nível de bairro, usando o Índice de Moran. Os mapas de correlação e significância podem ser observados na Figura 4
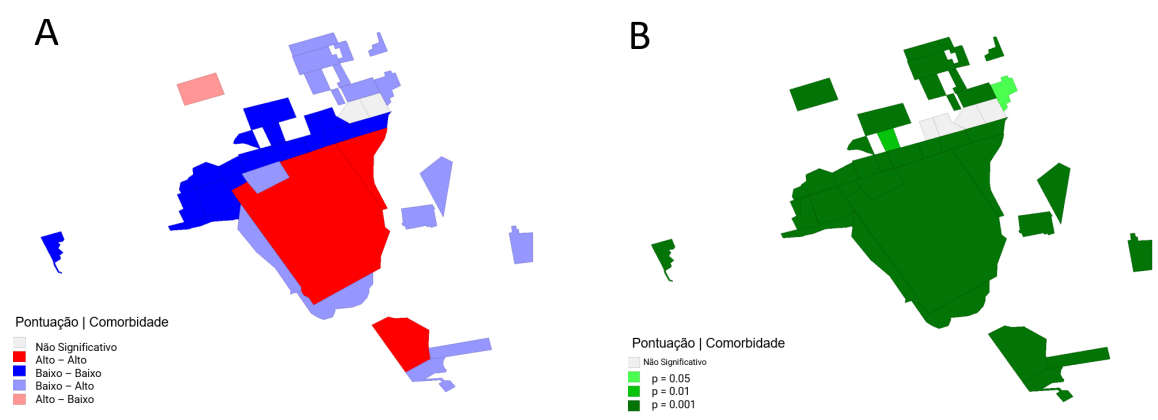

Figura 4: A - Análise do índice de Moran Local (LISA). B - Mapa de significância

Os valores para o índice de Moran variam de -1 a 1 , sendo que se $I>0$ significa que existe uma correlação espacial positiva, já quando $I<0$ uma correlação espacial negativa. No presente caso, obteve-se um $I=0,412$ o que indica uma autocorrelação espacial positiva. Isto é, o teste sugere que existe semelhança entre os valores dos casos com comorbidades e pontuação.

A Figura 4-A mostra a distribuição de clusters de associação espacial entre número de casos com comorbidades e pontuação. É possível observar zonas com a classe Alta-Alta (bairros com altas taxas de casos com comorbidades e com alta pontuação) localizados nas áreas central e sul da cidade. Uma zona Alto-Baixo mais ao norte da cidade determina uma área com uma alta 
pontuação, porém com baixos casos de comorbidade. Com o intuito de testar a significância estatística, a interferência foi baseada em 999 permutações. Nos testes de significância obteve-se um $p-$ valor $=0.001$, o que indica que o índice de Moran calculado é significativo.

\section{Conclusões}

Ao longo do trabalho foi possível aplicar satisfatoriamente as ferramentas propostas. Os resultados mostraram a evolução da doença ao longo de 9 meses e permitiram constatar algumas relações. Entre os meses de abril a outubro a disseminação do vírus ocorreu de forma gradual, entretanto novembro apresentou uma elevação considerável de casos. As influências deste evento podem estar relacionadas a questões como o efeito de casos positivos provenientes de viagens, parte dos casos terem ocorridos em zonas de grande densidade demográfica e o efeito fronteira com a cidade de Artigas. As medidas de controlem tiveram bons resultados no início da epidemia, entretanto as flexibilizações podem ter contribuído com a contaminação comunitária no fim de 2020.

Com base no teste de Moran verificou-se que as zonas centrais e sul foram as zonas onde mais ocorreram correlações entre comorbidades e pontuações de risco. Sendo assim, o trabaho forneceu informações importantes referentes à COVID-19. Essas constatações podem auxiliar na formulação de medidas direcionadas à zonas específicas, contribuindo para uma melhor gestão da crise de COVID-19.

\section{Agradecimentos}

Os autores agradecem à CAPES pelo suporte financeiro e à Secretaria Municipal de Saúde do Município de Quaraí pelas contribuições no desenvovilmento do trabalho.

\section{Referências}

[1] Alcântara, E., Mantovai J., Rotta, L., Park, E., Rodrigues, T., Carvalho, F. C., Filho, C. R.S. Investigating spatiotemporal patterns of the COVID-19 in São Paulo State, Brazil. In Geospatial Health, volume 15, 2020. DOI: 10.4081/gh.2020.925.

[2] Cordes, J., Castro, M. C. Spatial analysis of COVID-19 clusters and contextual factors in New York City. In Spatial and Spatio-temporal Epidemiology, volume 34, pages 100355, 2020. DOI: 10.1016/j.sste.2020.100355.

[3] Cori, A., Ferguson, N.M., Fraser, C.,Cauchemez S. A new framework and software to estimate time-varying reproduction numbers during epidemics. In American journal of epidemiology, volume 178, pages 1505-1512, 2013. DOI: 10.1093/aje/kwt133.

[4] Dharmaratne, S., Sudaraka, S., Abeyagunawardena, I., Manchanayake, K., Kothalawala, M., Gunathunga, W. Estimation of the basic reproduction number (R0) for the novel coronavirus disease in Sri Lanka. In Virology Journal, volume 17, pages 1-7, 2020. DOI: 10.1186/s12985020-01411-0.

[5] Franch-Pardo, I., Napoletano,B.M., Rosete-Verges, F., Billa, L. Spatial analysis and GIS in the study of COVID-19. A review. In Science of The Total Environment, volume 739, pages 140033, 2020. DOI: 10.1016/j.scitotenv.2020.140033. 
[6] Gerber, M. S. Predicting crime using Twitter and kernel density estimation. In Decision Support Systems, volume 61, pages 115-125, 2014. DOI: 10.1016/j.dss.2014.02.003.

[7] Hang, H., Wang, Y., Chung, H. T., Chen, C. J. Clinical characteristics of novel coronavirus disease 2019 (COVID-19). In newborns, infants and children. Pediatrics Neonatology, volume 61(2), pages 131-132, 2020. DOI: 10.1016/j.pedneo.2020.03.001.

[8] Kristan, M., Leonardis, A. Discriminative Kernel Density Estimator With Gaussian Kernels. In IEEE Transactions on Cybernetics, volume 44, pages 355-365, 2017. DOI: 10.1109/TCYB.2013.2255983.

[9] Mohamed, K., Rodríguez-Román, E., Rahmani, F.,, Zhang, H., Ivanovska, M., Makka, S. A., Joya, M., Makuku, R.,Islam, M. S.,Radwan, N.,Rahmah, L., Goda, R., Abarikwu, S. O., Shaw, M., Zoghi, S., Irtsyan, S., Ling, I., Cseprekal, O., Faten, O., Sayar, E.H., Soloukey, C.,Grancini, G., Rezaei, N. Borderless collaboration is needed for COVID-19-A disease that knows no borders. In Infection Control Hospital Epidemiology, volume 41, pages 1245-1246, 2020. DOI: $10.1017 /$ ice.2020.162.

[10] Nishura, H., Linton,N. M., Akhmetzhanov,A. R. Serial interval of novel coronavirus (COVID19) infections. In International journal of infectious diseases, volume 93, pages 284-286, 2020. DOI: $10.1016 /$ j.ijid.2020.02.060.

[11] Peng, Z., Wang, R., Liu, L.,Wu, H. Exploring urban spatial features of COVID-19 transmission in Wuhan based on social media data. In International Journal of Geo-Information, volume 9, chapter 6, pages 402, 2020. DOI: 10.3390/ijgi9060402.

[12] Saffari, T., Oyelola, A. A., Gaywan, E., Elfaki, F., Kuddus, M. A. Saffary, R. Analysis of COVID-19 cases' spatial dependence in US counties reveals health inequalities. In Frontiers in public health, volume 8, 2020. DOI: 10.3389/fpubh.2020.579190.

[13] Sanyaolu, A., Okorie, C., Marinkovic, A., Patidar, R., Younis, K., Desai, P., Hosein, Z., Padda, I., Mangat, J., Altaf, M. Comorbidity and its Impact on Patients with COVID-19. In comprehensive clinical medicine,volume 2, pages 1-8, 2020. DOI: 10.1007/s42399-020-00363-4.

[14] Scire, J., Nadeau, S.A., Vaughan, T et al. Reproductive number of the COVID-19 epidemic in Switzerland with a focus on the Cantons of Basel-Stadt and Basel-Landschaft. In Swiss medical weekly, volume 150, pages 20271, 2020. DOI: 10.3929/ethz-b-000415022.

[15] Shi, W., Tong,C.,Zhang, A., Wang, B., Shi, Z., Yao, Y., Peng, J. An extended Weight Kernel Density Estimation model forecasts COVID-19 onset risk and identifies spatiotemporal variations of lockdown effects in China. In Communications biology, volume 4, pages 1-10, 2021. DOI:10.1038/s42003-021-01677-2.

[16] Tonini, M., Pereira, G. M, Parente, J., Orozco, C. V. Evolution of forest fires in Portugal: from spatio-temporal point events to smoothed density maps. In Natural Hazards, volume 85,pages 1489-1510, 2017. DOI: 10.1007/s11069-016-2637-x.

[17] Wang D, Hu B, Hu C, Zhu F, Liu X, Zhang J, Wang B, Xiang H, Cheng Z, Xiong Y, Zhao Y, Li Y, Wang X, Peng Z. Clinical Characteristics of 138 Hospitalized Patients With 2019 Novel Coronavirus-Infected Pneumonia in Wuhan, China. In JAMA, volume 323, pages 1061-1069, 2020. DOI: $10.1001 /$ jama.2020.1585.

[18] Zhang Y., Wang, Y., Zhou, G., Jin, J., Wang, X., Cichocki, A. Multi-kernel extreme learning machine for EEG classification in brain-computer interfaces. In Expert Systems with Applications, volume 96, pages 302-310, 2018. DOI:10.1016/j.eswa.2017.12.015. 